\title{
A Study on Risk Factors and Etiology of Female Infertility
}

\author{
Lili Xuan, Xiaojun Liu, Jia Wang \\ China-Japan Union Hospital of Jilin University, Changchun, Jilin, 130033, China
}

Keywords: Infertility, Risk factors, Oviduct function etiology, Endocrine function etiology

\begin{abstract}
The paper investigated the related factors and causes of female infertility. The clinical data of 150 infertile women who were treated in China-Japan Union Hospital of Jilin University from January, 2015 to December, 2017 were analyzed retrospectively. The main influencing factors and causes of infertility were analyzed. The related factors of female infertility were tubal obstruction, endocrine factor, uterine factor, ovarian mass, cervix factor, pelvic inflammatory disease, immune factor, vaginal factor and endometriosis. Polycystic ovary syndrome with $41.4 \%$ percent, luteal insufficiency with 28.6 percent and excessive mental pressure with 21.4 percent were found in the patients with primary infertility. The endocrine causes of secondary infertility were polycystic ovary syndrome (PCOS) with 42.5 percent, luteal insufficiency with 28.8 percent and excessive mental stress with 21.3 percent. The etiology of fallopian tube function in patients with primary infertility was 38.6 percent in reproductive system and 30.0 percent in endometriosis. The etiology of fallopian tube function in secondary infertility was 38.8 percent in reproductive system and 31.3 percent in endometriosis. It can be concluded that the occurrence of infertility in female is affected by various factors, and the education in reproductive health should be strengthened continuously, so as to prevent the occurrence of infertility.
\end{abstract}

\section{Introduction}

Affected by the increasingly open social concept, the number of patients with sexually transmitted diseases is increasing, and the incidence of infertility is increasing year by year. According to a recent WHO study, the proportion of infertile couples among married couples has reached 7 to 15 percent, of which 50 to 60 percent are due to simple infertility caused by female factors. The incidence of female infertility in China is about 3.56 percent, and the etiology is various, which is related to the factors such as lifestyle, fallopian tube disease, endocrine disorder and so on, which has a great negative effect on female physical and mental health. In this paper, 150 cases of female infertility were selected as the research objects, and the risk factors of infertility and infertility were identified, so as to provide references for clinical diagnosis and treatment of the disease. The report is as follows.

\section{Materials and Methods}

\subsection{Clinical Data}

The clinical data of 150 infertile women who were treated in our hospital from January, 2015 to December, 2017 were analyzed retrospectively. Among them, 70 cases were primary infertility and 80 cases were secondary infertility. All the subjects were between 25 to 40 years old with an average age of $30.9 \pm 3.5$ years. The subjects excluded from the study were combined with serious cardiovascular, tumor and blood diseases, so as not to affect the results of the study.

\subsection{Methods}

The following studies were conducted in 150 infertile patients: First, communicate with the patients and understand their pregnancy history, surgical history, disease history, menstrual status and sexual behavior. Second, we have to perform gynecological examinations, and perform vaginal sonography to examine the vagina, uterus and its appendages, fallopian tubes, etc. In addition, we need to perform endocrine tests on follicle stimulating hormone, prolactin, luteinizing hormone, 
progesterone and so on. Third, we examined the patient's genital tract to see if there was an infection. Fourth, we performed an examination of the fluid of the fallopian tube. Fifth, we made sexual intercourse test. Sixth, we gave hysteroscopy to check the uterus and its appendages. Seventh, immunological examination was performed. After the above examinations, the related factors and causes of primary infertility and secondary infertility were statistically analyzed, providing a theoretical reference for clinical diagnosis and treatment.

\subsection{Statistical Method}

We used SPSS 21.0 software for data processing, measured data by $t$ test, and counted data by $\chi^{2}$ test. The difference was statistically significant $(\mathrm{P}<0.05)$.

\section{Results}

\subsection{Etiological Statistics of Patients with Primary and Secondary Infertility}

According to the statistical results of clinical data, female infertility is mainly related to tubal obstruction, endocrine factors, uterine factors, ovarian mass, cervical factors, pelvic inflammation, immune factors. Vaginal factors and endometriosis, including tubal obstruction and endocrine factors. (See in Table 1)

Table 1. Etiology Statistics of Patients with Primary and Secondary Infertility n (\%)

\begin{tabular}{ccc}
\hline Cause of Disease & Primary Infertility(n=70) & Secondary Infertility $(\mathrm{n}=80)$ \\
\hline Uterine Factor & $5(7.1)$ & $2(2.5)$ \\
Oviduct Obstructed & $15(21.4)$ & $24(30.0)$ \\
Ovarian Mass & $6(8.6)$ & $2(2.5)$ \\
Cervical Factors & $5(7.1)$ & $9(11.3)$ \\
Vaginal Factor & $6(8.60$ & $7(8.8)$ \\
Incretion & $15(21.4)$ & $17(21.3)$ \\
pelvic Inflammation & $5(7.1)$ & $1(1.3)$ \\
Immunological Factors & $4(5.7)$ & $5(6.3)$ \\
Endometriosis & $1(1.4)$ & $1(1.3)$ \\
\hline
\end{tabular}

\subsection{Statistics on the Causes of Fallopian Tube Function in Patients with Primary and Secondary Infertility}

The endocrine causes of primary infertility included polycystic ovary syndrome with 41.4 percent, luteal insufficiency with $28.6 \%$ and excessive mental stress with 21.44 percent. The endocrine causes of secondary infertility were polycystic ovary syndrome (PCOS) with 42.5 percent, luteal insufficiency with 28.8 percent and excessive stress with 21.3 percent. (See in Table 2)

Table 2. Statistics of the Causes of Fallopian Tube Function in Patients with Primary and Secondary Infertility n (\%)

\begin{tabular}{ccc}
\hline Reason in Fallopian Tube & Primary Infertility(n=70) & Secondary Infertility(n=80) \\
Function & $27(38.6)$ & $31(38.8)$ \\
Genital System Inflammation & $21(30.0)$ & $25(31.3)$ \\
Endometriosis & $13(18.6)$ & $14(17.5)$ \\
Abdominal Surgery Injury & $8(11.4)$ & $9(11.3)$ \\
Cacoepy &
\end{tabular}

\subsection{Statistics of Endocrine Causes in Patients with Primary and Secondary Infertility}

The etiology of fallopian tube function in patients with primary infertility was 38.6 percent in reproductive system and 30.0 percent in endometriosis. The etiology of fallopian tube function in secondary infertility was 38.8 percent in reproductive system and 31.3 percent in endometriosis. (See in Table 3) 
Table 3. Endocrine Causes in Patients with Primary and Secondary Infertility n (\%)

\begin{tabular}{ccc}
\hline Endocrine Etiology & Primary Infertility $(\mathrm{n}=70)$ & Secondary Infertility $(\mathrm{n}=80)$ \\
\hline Stein-Leventhal Syndrome & $29(41.4)$ & $34(42.5)$ \\
Luteal Phase Defect & $20(28.6)$ & $23(28.8)$ \\
Excessive Stress & $15(21.4)$ & $17(21.3)$ \\
Luteinized Follicular & $3(4.3)$ & $4(5.0)$ \\
Unruptured Syndrome & & $3(3.8)$ \\
Hyperprolactinemia & $2(2.9)$ & \\
\hline
\end{tabular}

\section{Discussions}

Pregnancy is a complex physiological process. Generally speaking, pregnancy requires the following conditions: If the female reproductive system develops normally and has a normal sex life, and the follicle matures after ovulation begins; sperms enter the vagina and meet the mature egg in the fallopian tube. The head of the sperm is in contact with the surface of the egg, and the fertilization begins, after which the fertilized egg enters the uterine chamber and develops. Infertility in women is one of the diseases endangering human reproductive health, which involves the reproductive health of couples of childbearing age in all countries and regions of the world. Although it is not a fatal disease, it causes a series of physical and psychological injuries to patients and families, and even causes some marriages to break up. This is a major medical and social problem all over the world. How to solve the problem of infertility is the unshirkable responsibility of medical workers. The etiology of infertility in women is very complex. Any factor that affects the ovulatory "fertilization" implantation can cause disease, and the factors often affect each other. In this study, in primary infertility and secondary infertility, fallopian tube failure and endocrine disorders accounted for the largest proportion of various factors. Analysis of reasons: The tubal impassability often causes the tubal cavity adhesion "stiff" because of the pelvic chronic inflammation, or is pulled by the surrounding scar tissue distortion or the occlusion, causing the fallopian tube to lose its transport sperm "egg" the pregnant egg physiological function causing infertility; endocrine mainly includes polycystic ovary syndrome, luteal dysfunction, excessive stress, luteinized follicular unruptured syndrome and hyperprolactinemia.

The data of this study showed that the related factors of female infertility are mainly including tubal obstruction, endocrine factors, uterine factors, ovarian mass, cervix factor, pelvic inflammatory disease, immune factors, vaginal factors and endometriosis and other factors. Tubal obstruction and endocrine-related diseases are the highest proportion of causes. In the endocrine aspect, the endocrine causes of primary infertility were as follows: polycystic ovary syndrome (PCOS) accounted for 41.4 percent, luteal insufficiency accounted for 28.6 percent and mental stress accounted for 21.4 percent. The endocrine causes of secondary infertility were polycystic ovary syndrome (PCOS) with 42.5 percent, luteal insufficiency accounted for 28.8 percent and excessive mental stress accpunted for 21.3 percent. The main manifestations of tubal impassability were as follows: polycystic ovary syndrome (PCOS) accounted for 41.4 percent, luteal insufficiency accounted for 28.6 percent, and excessive mental stress accounted for 21.4 percent in primary infertility. The endocrine causes of secondary infertility were polycystic ovary syndrome (PCOS) with 42.5 percent, luteal insufficiency accounted for 28.8 percent and excessive mental stress was 21.3 percent. The above research data are basically consistent with the conclusions of other domestic scholars.

According to the results of this study, the main etiology of infertile women in addition to physiological diseases, mental and psychological factors also have a very significant impact. Infertility is not only a problem of reproductive health, but also a social problem. Studies have shown that mental factors can cause infertility. The old ideas passed down in rural areas of our country still have a great impact. Infertility causes mental tension among couples through many 
ways. Some infertile couples are under great mental pressure, and even have a sense of inferiority complex and shame. This problem seriously affects the quality of life of the husband and wife, which is influenced by traditional ideas in the absence of a clear cause of infertility. The psychological stress of female patients is also much higher than that of male patients. We should pay attention to the mental health of female infertile patients and strengthen psychological guidance in order to improve their psychological state and increase the rate of pregnancy.

To sum up, infertility is a common disease in women, and the etiology is more. Identifying the related risk factors and etiology can point out the direction of clinical treatment and prevention and reduce the incidence. The sample size of this study is limited, and further research is needed for the possible influencing factors of infertility.

\section{References}

[1] Chen Yinhua. Study on the Related Factors and Etiology of Female Infertility [J]. Shenzhen Journal of Integrated Chinese and Western Medicine: 07: 77-78.

[2] Huang Jie. Analysis of Related Factors and Etiology of 296 Cases of Female Infertility [J]. Heilongjiang Medical Science: 01: 142-144.

[3] Li Ailing. Etiological Analysis of 200 Cases of Female Infertility [J]. China Disability Medicine, 2013: 07: 417-418.

[4] Zhao Yongxin. Analysis of Related Factors and Etiology of Female Infertility in 980 cases [J]. Modern Preventive Medicine 14: 3528-3529.

[5] Xi Bo. Causes and Preventive Measures of Infertility [J]. Inner Mongolia Traditional Chinese Medicine (TCM): 12: 135. 